\section{Pneumoniediagnostik in der Hausarztpraxis: Gibt es Frühwarnzeichen?}

Moore $\mathrm{M}$ et al. Predictors of pneumonia in lower respiratory tract infections: $3 C$ prospective cough complication cohort study. Eur Respir ] 2017; 50: 1700434

Infekte der unteren Atemwege zählen zu den häufigsten Konsultationsanlässen in der Hausarztpraxis. Eine Pneumonie entwickelt sich im Verhältnis eher selten, kann aber unerkannt und unbehandelt schwere Folgen nach sich ziehen. Moore und Kolleginnen/Kollegen wollten nun herausfinden, welche klinischen Charakteristika und Befunde zu Beginn einer Bronchitis als Prädiktoren einer sekundären Pneumonie geeignet sind. Dazu initiierten sie eine prospektive Kohortenstudie mit über 28000 Patientinnen und Patienten.

Besonders in der kalten Jahreszeit leiden viele Menschen unter Erkältungssymptomen wie Husten und Schnupfen, und stellen sich mit Infekten der unteren Atemwege bei ihrer Hausärztin bzw. bei ihrem Hausarzt vor. Erhielten früher noch viele von ihnen umgehend eine antibiotische Behandlung, ist der Einsatz dieser „Wunderwaffe“ gegen Keime vor dem Hintergrund zunehmender Resistenzentwicklungen heute deutlich restriktiver geworden. Nicht alle Patientinnen/Patienten haben eine schwere bakterielle Infektion, nur wenige von ihnen entwickeln im Verlauf die Komplikation einer Lungenentzündung. Um mögliche Faktoren ausfindig machen zu können, die sich zum Zeitpunkt der ersten Konsultation als Prädiktoren für die Progression zur Pneumonie eignen, haben Moore und sein Team den Krankheitsverlauf von insgesamt 28883 erwachsenen Patientinnen und Patienten auf Ebene der primärärztlichen Versorgung unter die Lupe genommen. Alle stellten sich mit einem neu aufgetretenen Infekt der unteren Atemwege, einer Bronchitis, bei ihren Hausärztinnen/Hausärzten vor. Sie wurden aus 5333 Praxen des Vereinigten Königreiches rekrutiert und nach der ersten Konsultation für 30 Tage nachbeobachtet. Unter 720 Patientinnen und $\mathrm{Pa}$ - tienten, die innerhalb der ersten Woche eine Röntgen-Thorax-Untersuchung erhielten, suchten die Autorinnen/Autoren diejenigen heraus, bei denen die Diagnose einer Pneumonie sicher oder wahrscheinlich war. Zur Detektion von Prädiktoren verglichen Moore und sein Team schließlich die klinischen Charakteristika und Befunde vom ersten Arztbesuch mit den entsprechenden Daten der Patientinnen/Patienten ohne Pneumonie. Einschlusskriterien waren:

- Alter über 16 Jahre,

- neu aufgetretene Erkrankung,

- Symptome mit Husten,

- Infektionszeichen nach Beurteilung des behandelnden Arztes.

Ausschlusskriterien waren andere Ursachen des Hustens wie Herzinsuffizienz oder chronische Lungenerkrankungen. Es wurden folgende Parameter erhoben:

- Alter,

- Symptome und Symptomverlauf,

- klinischer Untersuchungsbefund,

- Schwere der Krankheit.

In die Kategorie „klinischer Untersuchungsbefund“ entfielen die Parameter Auskultationsbefund, Temperatur, Sauerstoffsättig, Herzfrequenz und Blutdruck. Die Krankheitsschwere konnte mit Hilfe einer visuellen Analogskala (VAS) von „gut“ bis „sehr schlecht“ eingeschätzt werden. Zur statistischen Auswertung rechneten die Autorinnen/Autoren eine logistische Regressionsanalyse.

\section{Erhöhte Temperatur als stärkster Prädiktor}

Von 1782 Patientinnen und Patienten konnten die Untersucherinnen/Untersucher auf Röntgenbilder zurückgreifen, bei 720 davon fand die Diagnostik innerhalb der ersten Woche nach der ersten Konsultation beim Hausarzt statt. Eine Pneumonie stellten die Autorinnen/Autoren in 115 Fällen fest. Im direkten Vergleich mit Patientinnen und Patienten ohne Pneumonie-Diagnose im Verlauf gab es im Bereich der klinischen Charakteristika nur minimale Gruppenunterschiede. Diese erwiesen sich in der logistischen Regression als ungeeignet, um eine Pneumonie vorhersagen zu können. Ganz anders verhielt es sich bei den klinischen Untersuchungsbefunden. Hier erwiesen sich die folgenden Messwerte als schlagkräftige Prädiktoren:

- Körpertemperatur über $37,8^{\circ} \mathrm{C}$,

- Rasselgeräusche als Auskultationsbefund,

- Tachykardie mit einer Herzfrequenz über 100/Minute,

- Sauerstoffsättigung unter $95 \%$.

War bereits die Körpertemperatur in der Sensitivitätsanalyse ein bedeutsamer Prädiktor, konnte der Vorhersagewert mit Hinzunahme der anderen 3 Parameter noch gesteigert werden. Für den Blutdruck konnten die Untersucherinnen und Untersucher einen solchen Effekt nicht feststellen.

FAZIT

Eine erhöhte Körpertemperatur von über $37,8^{\circ} \mathrm{C}$ war der stärkste Prädiktor für die Entwicklung einer Pneumonie als Komplikation von Infektionen der unteren Atemwege. Unter Berücksichtigung des Auskultationsbefundes, der Herzfrequenz sowie der Sauerstoffsättigung konnte der Vorhersagewert noch gesteigert werden. Die Autorinnen/Autoren betonen daher die Bedeutung einer gründlichen klinischen Untersuchung und empfehlen eine routinemäßige pulsoxymetrische Messung der Sauerstoffsättigung in der Hausarztpraxis.

Dipl.-Psych. Annika Simon, Hannover 